\title{
Erratum to: Impact of hemoglobin levels on renal and non-renal clinical outcomes differs by chronic kidney disease stages: the Gonryo study
}

Tae Yamamoto $^{1} \cdot$ Mariko Miyazaki $^{1,2} \cdot$ Masaaki Nakayama $^{2,3} \cdot$ Gen Yamada $^{1}$. Masato Matsushima ${ }^{2,4} \cdot$ Mistuhiro Sato $^{5}$ - Toshinobu Sato ${ }^{5}$ - Yoshio Taguma ${ }^{2,5}$. Hiroshi Sato ${ }^{2,6} \cdot$ Sadayoshi Ito ${ }^{1,2}$

Published online: 26 June 2017

(C) Japanese Society of Nephrology 2017

\section{Erratum to: Clin Exp Nephrol (2016) 20:595-602 DOI 10.1007/s10157-015-1190-3}

In the original publication Fig. 1 was incorrectly published. The revised version of Fig. 1 is given in this Erratum.

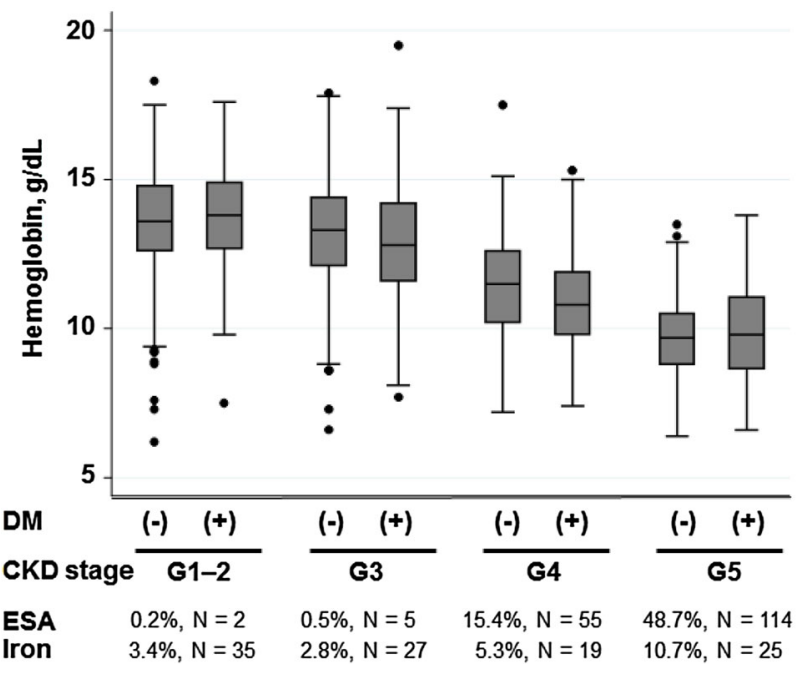

The online version of the original article can be found under doi:10.1007/s10157-015-1190-3.

Tae Yamamoto

tae.yamamoto@med.tohoku.ac.jp

1 Division of Nephrology, Endocrinology and Vascular Medicine, Tohoku University Graduate School of Medicine, 1-1 Seiryomachi, Aoba-ku, Sendai, Miyagi 980-8574, Japan

2 Center for Advanced Integrated Renal Science, Tohoku University Graduate School of Medicine, Sendai, Japan

3 Division of Nephrology, Hypertension, Diabetology, Endocrinology, and Metabolism, Fukushima Medical University School of Medicine, Fukushima, Japan

4 Department of Clinical Research, The Jikei University School of Medicine, Tokyo, Japan

5 Kidney Center, Japan Community Health Care Organization Sendai Hospital, Sendai, Japan

6 Clinical Pharmacology and Therapeutics, Tohoku University Graduate School of Pharmaceutical Sciences, Sendai, Japan 